BMJ Open

Diabetes

Research

\& Care

\section{Improving interpretability of individual Diabetes Symptom Checklist-Revised (DSC-R) scores: the role of patient characteristics}

To cite: Wieringa TH, de Wit M, Twisk JWR, et al. Improving interpretability of individual Diabetes Symptom ChecklistRevised (DSC-R) scores: the role of patient characteristics. BMJ Open Diab Res Care 2020;8:e001146. doi:10.1136/ bmjdrc-2019-001146

Received 22 December 2019 Revised 2 March 2020 Accepted 9 March 2020

\section{Check for updates}

(C) Author(s) (or their employer(s)) 2020. Re-use permitted under CC BY-NC. No commercial re-use. See rights and permissions. Published by BMJ.

${ }^{1}$ Department of Medical Psychology, Amsterdam UMC, Vrije Universiteit, Amsterdam Public Health research institute, Amsterdam, The Netherlands ${ }^{2}$ Department of Epidemiology and Biostatistics, Amsterdam UMC, Vrije Universiteit, Amsterdam Public Health research institute, Amsterdam, The Netherlands

Correspondence to Dr Thomas H Wieringa; t.wieringa@amsterdamumc.nl

\section{ABSTRACT}

Introduction The Diabetes Symptom Checklist-Revised (DSC-R) is a well-validated patient-reported outcome designed to assess symptom burden in persons with type 2 diabetes mellitus (T2DM) across eight domains. The DSC-R has so far primarily been used in research settings. With the aim to make the DSC-R applicable in clinical practice by improving its interpretability, we sought to identify patient characteristics associated with DSC-R (domain) scores as a first initiative toward reference values.

Research design and methods We used baseline data from two large observational studies to select patient characteristics significantly associated with DSC-R domain and total scores. Multivariable Tobit analyses with the backward procedure per (domain) score were performed. Results Data from 1531 participants with T2DM were included. On a 0-100 scale, the median DSC-R total score was 15.88 (7.06-29.41), with domain scores ranging from 5.00 (0.00-22.50) (pain) to 35.00 (10.00-60.00) (fatigue). Low well-being status was most profoundly associated with higher scores across all domains. Persons with one or more complication, as well as one or more symptomatic hypoglycemic episode during the past 3 months, scored higher on (almost) all domains and the total scale.

Conclusions Complications, symptomatic hypoglycemia, and low well-being are important characteristics to take into account when using the DSC-R in individual patients. Further validation of our findings is warranted in diverse patient populations.

\section{BACKGROUND}

The Diabetes Symptom Checklist (DSC) was developed by Grootenhuis et $a l^{1}$ almost 25 years ago in the context of the Hoorn study to reliably capture the experience of diabetesrelated symptom distress of persons with type 2 diabetes mellitus (T2DM) and changes therein as a result of medical treatment. ${ }^{1}$ Based on research data, the DSC was revised in two ways: (1) for the sake of simplicity and to avoid confusion, the frequency scale was replaced by a dichotomous yes/no response for the presence or absence of each symptom; and (2) the scaling was changed from a

\section{Significance of this study}

What is already known about this subject?

- The Diabetes Symptom Checklist-Revised (DSC-R) is a well-validated, widely used patient-reported outcome designed to assess symptom burden in persons with type 2 diabetes mellitus across eight domains.

- The DSC-R has so far primarily been used in research settings and may have clinical utility.

- Individual use of DSC-R scores in routine care requires good interpretability, based on reference values.

What are the new findings?

- Diabetes complications, symptomatic hypoglycemia, and low well-being are characteristics to take into account when using the DSC-R in individual patients.

How might these results change the focus of research or clinical practice?

- The relevant associations presented and their directions can help improve the interpretability of DCS-R domain and total scores.

- Especially mood status should be taken into account.

- The associations found may be a first step for future research to focus on creating reference values or weights for different groups, as well as establishing clinically meaningful differences in diabetes symptom burden.

4-point to a 5-point Likert scale to enhance variability, ${ }^{2}$ resulting in the DSC-Revised (DSC-R). ${ }^{3}$ The DSC-R consists of 34 items grouped into 8 symptom domains: fatigue, cognitive symptoms, pain, sensitivity symptoms, cardiological symptoms, ophthalmic symptoms, hypoglycemia, and hyperglycemia. It asks about the burden of diabetes symptoms experienced during the past month. The DSC-R has good psychometric properties $^{3}$ and has been validated in a multitude of languages and used primarily as patientreported outcome (PRO) in clinical trials. 


\begin{tabular}{|c|c|}
\hline \multicolumn{2}{|l|}{ Gender } \\
\hline Female & 750 (49.20\%) \\
\hline Age & $61.37(10.90)$ \\
\hline \multicolumn{2}{|l|}{ Educational level } \\
\hline Low & $699(53.60 \%)$ \\
\hline Middle & 467 (35.80\%) \\
\hline High & $138(10.60 \%)$ \\
\hline Diabetes duration (years) & $7.00(4.00-12.00)$ \\
\hline \multicolumn{2}{|l|}{ Complications } \\
\hline 0 & $881(62.70 \%)$ \\
\hline$\geq 1$ & $523(37.30 \%)$ \\
\hline \multicolumn{2}{|l|}{ Comorbidities } \\
\hline 0 & $1367(89.30 \%)$ \\
\hline$\geq 1$ & $164(10.70 \%)$ \\
\hline \multicolumn{2}{|l|}{$\mathrm{HbA1c}$} \\
\hline $\mathrm{mmol} / \mathrm{mol}$ & $69.32(16.45)$ \\
\hline$\%$ & $8.49(1.51)$ \\
\hline Body mass index & $30.53(6.27)$ \\
\hline \multicolumn{2}{|l|}{ Treatment } \\
\hline Oral agents & $1021(66.70 \%)$ \\
\hline Insulin & $510(33.30 \%)$ \\
\hline \multicolumn{2}{|c|}{$\begin{array}{l}\text { Symptomatic hypoglycemia during the past } 3 \text { months (self- } \\
\text { report) }\end{array}$} \\
\hline 0 episode & $584(48.90 \%)$ \\
\hline$\geq 1$ episode & $610(51.10 \%)$ \\
\hline \multicolumn{2}{|c|}{ Severe hypoglycemia during the past 3 months (self-report) } \\
\hline 0 episode & $1191(94.30 \%)$ \\
\hline$\geq 1$ episode & $72(5.70 \%)$ \\
\hline WHO-5 score (well-being) & $60.00(40.00-76.00$ \\
\hline
\end{tabular}

*Based on non-imputed data.

†For categorical variables: frequencies (valid percentages); for normally distributed continuous variables: mean (SD); for skewed distributed continuous variables: median (25th-75th percentile). $\mathrm{HbA1c}$, hemoglobin A1c.

When aiming to use the DSC-R as PRO in clinical practice, reference values are an important feature to consider. Interpretability is a key issue for using the DSC-R in clinical practice, that is, in individual patients, and can be defined as 'the degree to which one can assign qualitative meaning to an instrument's quantitative scores or change in scores', or in other words 'the degree to which it is clear what the scores or change scores mean'. ${ }^{2}$ Interpretability is not a measurement property, like validity and reliability, because it does not refer to the quality of an instrument. Rather, it refers to what the scores on an instrument mean and is a prerequisite for any instrument to be applicable in clinical practice. In this context it is essential to have reference values, ${ }^{2}$ differentiated according to relevant patient characteristics. For example, previous research has shown that symptom report is partly explained by negative affect. ${ }^{4-6}$ In the Hoorn screening study, negative mood was found to significantly amplify diabetes symptom burden, as measured by the DSC-R. ${ }^{5}$ In other words, when interpreting DSC-R scores on an individual basis, we need to be recognizant of patient-related factors that may influence symptom reporting, such as gender, age, and complication status, and these associations may be generic or domain-specific. For this purpose we need to assess which patient characteristics are associated with DCS-R domain and total scores.

The current study aims to improve the clinical usefulness of the DSC-R through establishing which patient characteristics are associated with DSC-R (domain) scores.

\section{METHODS}

Baseline data were used from the SPIRIT (Study of the Psychological Impact in Real care of Initiating insulin glargine Treatment) ${ }^{7}$ and the ESPRIT (Effect Study on Patient-Reported outcomes in Insulin glargine Treatment $)^{8}$ studies and were merged. The SPIRIT data set includes data from 1021 persons with T2DM prior to switching from oral glucose-lowering agents to a longacting insulin (glargine-100). The ESPRIT data set includes 510 persons with T2DM prior to switching from any long-acting insulin to insulin glargine-100. Details of the SPIRIT and the ESPRIT study are reported elsewhere. $^{78}$

In both SPIRIT and ESPRIT, hemoglobin A1c (HbA1c) was retrieved from the medical chart and demographic and clinical data were self-reported. ${ }^{78}$ The DSC-R and the WHO-5 Well-Being Index were completed and used in the current study. The WHO-5 Well-Being Index consists of five positively worded items assessing emotional wellbeing pertaining to the past 2 weeks. ${ }^{9}$ Scores are transformed to $0-100$, with higher scores representing better emotional well-being. ${ }^{9}{ }^{10}$ Scores were divided into categories: a score $\leq 28$ is indicative of depression, ${ }^{11}$ a score $>28$ and $\leq 50$ is indicative of low mood, ${ }^{10} 12$ and a score higher than 50 is indicative of normal well-being.

\section{Analyses}

Multiple imputation on the item level was performed, in which imputation models were created per DSC-R domain score. These imputation models contained items of the domain, as well as the original (non-dichotomized) patient characteristics potentially associated with the DSC-R (domain) scores. Multiple imputation using five imputations, which results in five imputed data sets, was performed in SPSS V.22.

Both DSC-R domain and total scores were standardized to $0-100$ scores, with higher scores representing higher symptom burden. Because of the large numbers of zero-scores for the DSC-R domains and total scale, Tobit regression analyses were performed using Stata V.15. ${ }^{13}$ All analyses were repeated in five different data sets and consisted of three steps: (1) multivariable Tobit 
Table 2 Median and IQR for DSC-R total scores and domain scores $(n=1531)^{\star}$

\begin{tabular}{lr}
\hline Total DSC-R & $15.88(7.06-29.41)$ \\
\hline Fatigue & $35.00(10.00-60.00)$ \\
\hline Cognitive symptoms & $15.00(0.00-40.00)$ \\
\hline Pain & $5.00(0.00-22.50)$ \\
Sensitivity symptoms & $6.67(0.00-26.67)$ \\
Cardiological symptoms & $10.00(0.00-25.00)$ \\
Ophthalmic symptoms & $8.00(0.00-24.00)$ \\
Hypoglycemia & $6.67(0.00-26.67)$ \\
Hyperglycemia & $20.00(5.00-40.00)$ \\
\hline
\end{tabular}

*Based on non-imputed (original) data.

DSC-R, Diabetes Symptom Checklist-Revised.

analyses using a backward procedure to select the characteristics significantly associated with the domain scores and total DSC-R score ${ }^{14}$; (2) final models were created only for those variables significantly associated with the outcome of interest in at least three imputed data sets; and (3) based on the final models, Rubin's rule was used to obtain pooled regression coefficients and 95\% CIs. A $\mathrm{p}$ value of 0.05 was used as threshold for a statistically significant association.

Patient characteristics potentially associated with DSC-R (domain) scores were dichotomized in order to enhance interpretability and clinical applicability based on medians and guidelines. ${ }^{15} 16$ The following were the variables found to be associated with symptom burden in previous studies and were included as independent variables in the first model for the backward procedure:

- Sociodemographics: gender, age $(<70$ years vs $\geq 70$ years), and level of education (low, middle, high).

- Clinical characteristics: diabetes duration $(<10$ years vs $\geq 10$ years), complication status ( 0 vs $\geq 1$ ), comorbidity $(0$ vs $\geq 1)$, glycemic control (HbAlc; $\leq 64.00 \mathrm{mmol} /$ $\mathrm{mol}(\leq 8.00 \%)$ vs $>64.00 \mathrm{mmol} / \mathrm{mol}(>8.00 \%))$, body mass index $(\mathrm{BMI})$ (non-obese $(<30)$ vs obese $(\geq 30)$ ), treatment (using oral agents vs using insulin), selfreported symptomatic hypoglycemia ( 0 vs $\geq 1$ episode in the past 3 months), and self-reported severe hypoglycemia ( 0 vs $\geq 1$ episode in the past 3 months).

- Psychological well-being status (normal well-being, low mood, likely depression). ${ }^{35} 6$ 17-22

\section{RESULTS}

The total data set included 1531 patients with T2DM, of whom $49.20 \%$ were female and with a mean diabetes duration of 7 years (table 1 ).

The median and IQR (25th-75th percentile) for the DSC-R domain and total scores of the study population are presented in table 2. The median DSC-R total score was 15.88 (7.06-29.41), and the median domain scores ranged from $5.00(0.00-22.50)$ (pain) to 35.00 (10.0060.00) (fatigue).

\section{Tobit analyses}

Patient characteristics that were significantly associated with DSC-R scores are presented in table 3. Persons with a diabetes duration of $\geq 10$ years report less burden of fatigue, cognitive symptoms, and hyperglycemia, as well as total burden, compared with those with shorter disease duration. Suffering from one or more complication was associated with a higher total score as well as higher scores on all DSC-R domains, except for the hypoglycemia domain. Reporting one or more symptomatic hypoglycemic episode was found to be significantly associated not only with higher hypoglycemia symptom burden, but also with higher scores on all other domains. Lower well-being status (both low vs normal and likely depression vs normal) showed to be strongly associated with higher scores for all DSC-R domains and the total score.

\section{DISCUSSION}

Based on combined data from two large observational studies including insulin-naïve and insulin-treated patients with T2DM, we investigated which patient characteristics are associated with patient-reported diabetes symptom burden. Responses on the DSC-R showed a wide variation in occurrence and degree of troublesomeness, underscoring the need to better understand interindividual differences, taking patient characteristics into account.

Fatigue is reported as the most common and most burdensome symptom of diabetes. Indeed, fatigue is known to be prevalent in persons with type 1 and type 2 diabetes. ${ }^{23-25}$ Fatigue was most pronounced in patients with lower well-being status. Persons with low mood score around 33 points (on a $0-100$ scale) higher compared with persons with normal well-being, while those likely depressed score approximately 46 points as higher relative to normal well-being. Low mood and likely depression do not only impact on fatigue, but amplify scores on all other domains of the DSC-R, in particular cognitive symptoms and hypoglycemic and hyperglycemic symptoms. Our findings are consistent with previous studies that found an association between psychological well-being and subjective symptom report. ${ }^{4-6}$ Several plausible explanations for this association have been suggested, but the causation remains unclear. Painful symptoms may induce or further increase depressed mood, ${ }^{26}$ while depression can amplify reported symptom burden, possibly due to a focus on symptoms ${ }^{27}$ and selective recall of negative events. ${ }^{28}$ Furthermore, negative affect may induce hypervigilance, which leads to an increase in 'scanning' of the body, that is, attention directed to the body, resulting in more somatic symptoms being detected. ${ }^{29}$ This mechanism may also drive the association between self-reported symptomatic hypoglycemia and DSC-R scores. ${ }^{19}$ Future research should aim to clarify this relationship by using continuous glucose monitoring for objective recording of hypoglycemic episodes. 


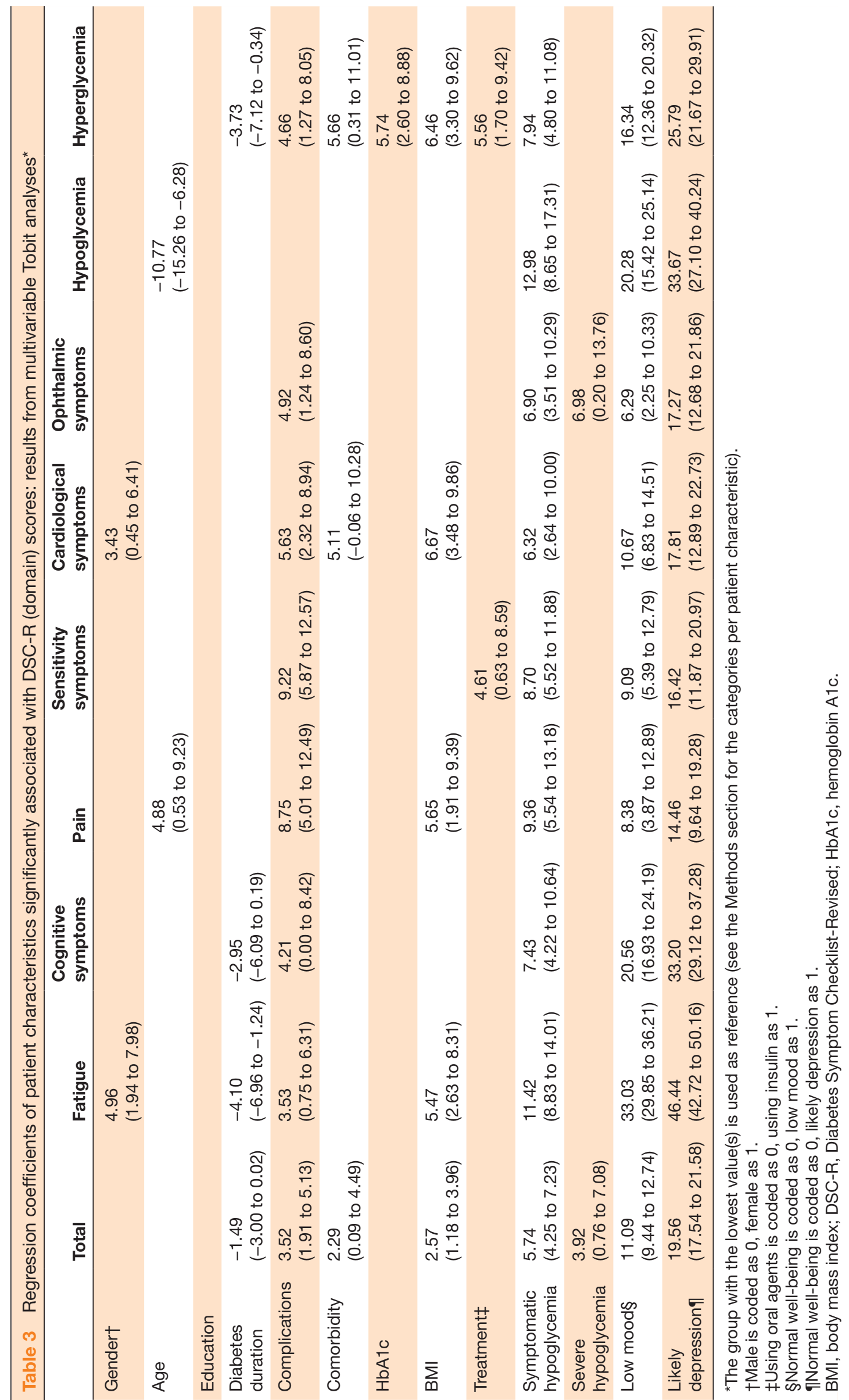


It is unclear why patients with a diabetes duration of $\geq 10$ years report lower fatigue, cognitive, hyperglycemia, and total symptom burden relative to those with shorter disease duration. Response shift or adaptation may play a role in this. ${ }^{2}$ Possibly, people suffering longer from diabetes may be less emotionally burdened compared with those recently diagnosed, resulting in lower negative affectivity in the latter group. Further research into the role of age and diabetes duration as a determinant of symptom distress is warranted.

Besides symptomatic hypoglycemia and diabetes duration, important clinical characteristics to take into account seem to be complication status and BMI. Interestingly, treatment regimen and glycemic control seem to differentiate less in terms of symptom burden. The strength of the association is probably dependent on the level of glycemic control, where one could expect a stronger impact on symptom burden in patients in poorer control versus those in better control. ${ }^{22}$

The significant associations and their regression coefficients presented here need further testing, but should help clinicians to interpret DCS-R domain and total scores, taking relevant patient characteristic into account. As to the clinical application of our findings, it is advised to focus on (changes in) DSC-R scores at the domain level. ${ }^{2}$ The total DSC-R score is informative, but we should be aware that no difference in total DSC-R score over time does not exclude the possibility that there actually might have been changes within domains (eg, one domain score worsened while another improved). Further research into the minimal clinically important difference (MCID) for the DSC-R is warranted for interpretation of changes in scores, building on a previous study providing preliminary results. ${ }^{3}$ The MCID is the smallest benefit of value to persons with T2DM capturing both the magnitude of the improvement and the value persons place on the change. ${ }^{30}$

\section{Strengths and limitations}

The data were derived from a large sample of persons with T2DM from both primary and secondary care settings at different stages of (insulin) therapy across different regions of the Netherlands, ${ }^{78}$ which favors the external validity (ie, generalizability) of our findings.

We were unable to study the role of different kinds of complications and comorbidities in symptom burden because of the relatively low prevalence of complications and comorbidities. This is a limitation of the current study as symptoms associated with T2DM may be directly related to complications and comorbidities. In this way, symptom burden domains are likely to be affected differently, depending on the seriousness and impact of complications and comorbidities. Furthermore, the relevant associations were found in a sample of mainly Caucasian patients with T2DM. Future research should replicate our study in diverse patient populations to define and further validate reference values. Here, studying the role of different kinds of complications and comorbidities will be of value. The relatively large number of missing data is a potential weakness of observational studies and was confirmed in the current study. However, multiple imputation can be viewed as the most robust way of dealing with missing data. ${ }^{31}$

\section{CONCLUSIONS}

The relevant associations presented and their directions can help improve the interpretability of the DCS-R domain and total scores. Future research may focus on creating reference values or weights for different patient groups, as well as establishing clinically meaningful differences in diabetes symptom burden.

Acknowledgements We would like to thank the Amsterdam Public Health research institute for providing a travel grant through which THW visited the Mayo Clinic in Rochester (Minnesota, USA). We would also like to thank the Knowledge and Evaluation Research Unit of the Mayo Clinic for sharing their ideas and giving suggestions regarding improvement of the DSC-R. We would like to thank Marcel Adriaanse for his time and effort searching data sets in which the DSC-R is included.

Contributors THW, MW, and FJS designed the study. THW performed the data analyses and drafted the manuscript. JWRT supported the data analyses. MW, JWRT, and FJS provided feedback on the manuscript (drafts), which was processed by THW. All authors read and approved the final manuscript.

Funding The authors have not declared a specific grant for this research from any funding agency in the public, commercial or not-for-profit sectors.

Competing interests The data reported here were collected from previous studies conducted with a grant from Sanofi received by the institution (Amsterdam UMC, location VU University Medical Center).

Patient consent for publication Not required.

Ethics approval The current study is based on two existing data sets. Both studies were, in view of their observational and non-invasive nature, not subject to the Dutch Medical Research Involving Human Subjects Act.

Provenance and peer review Not commissioned; externally peer reviewed.

Data availability statement The de-identified participant data that underlie the results reported in this article are available from m.dewit@amsterdamumc.nl upon reasonable request to researchers who provide a methodological sound proposal. Other documents that are available are study protocols and analytic codes. Proposals may be submitted up to 24 months following article publication.

Open access This is an open access article distributed in accordance with the Creative Commons Attribution Non Commercial (CC BY-NC 4.0) license, which permits others to distribute, remix, adapt, build upon this work non-commercially, and license their derivative works on different terms, provided the original work is properly cited, appropriate credit is given, any changes made indicated, and the use is non-commercial. See: http://creativecommons.org/licenses/by-nc/4.0/.

ORCID iD

Thomas H Wieringa http://orcid.org/0000-0001-8440-8844

\section{REFERENCES}

1 Grootenhuis PA, Snoek FJ, Heine RJ, et al. Development of a type 2 diabetes symptom checklist: a measure of symptom severity. Diabet Med 1994;11:253-61.

2 De Vet HCW, Terwee CB, Mokkink LB, et al. Measurement in medicine: a practical guide. 1st edn. Cambridge: Cambridge University Press, 2011.

3 Arbuckle RA, Humphrey L, Vardeva K, et al. Psychometric evaluation of the Diabetes Symptom Checklist-Revised (DSC-R)--a measure of symptom distress. Value Health 2009;12:1168-75.

4 Ciechanowski PS, Katon WJ, Russo JE, et al. The relationship of depressive symptoms to symptom reporting, self-care and glucose control in diabetes. Gen Hosp Psychiatry 2003;25:246-52.

5 Adriaanse MC, Dekker JM, Spijkerman AMW, et al. DiabetesRelated symptoms and negative mood in participants of a targeted 
population-screening program for type 2 diabetes: the Hoorn screening study. Qual Life Res 2005;14:1501-9.

6 Ludman EJ, Katon W, Russo J, et al. Depression and diabetes symptom burden. Gen Hosp Psychiatry 2004;26:430-6.

7 Hajos TRS, Pouwer F, de Grooth R, et al. Initiation of insulin Glargine in patients with type 2 diabetes in suboptimal glycaemic control positively impacts health-related quality of life. A prospective cohort study in primary care. Diabet Med 2011;28:1096-102.

8 Hajos TRS, Pouwer F, de Grooth R, et al. The longitudinal association between glycaemic control and health-related quality of life following insulin therapy optimisation in type 2 diabetes patients. A prospective observational study in secondary care. Qual Life Res 2012;21:1359-65.

9 World Health Organization. Wellbeing measures in primary health care: the DepCare project: report on a who meeting. Stockholm: WHO, 1998.

10 de Wit M, Pouwer F, Gemke RJBJ, et al. Validation of the WHO-5 well-being index in adolescents with type 1 diabetes. Diabetes Care 2007;30:2003-6.

11 Löwe B, Spitzer RL, Gräfe K, et al. Comparative validity of three screening questionnaires for DSM-IV depressive disorders and physicians' diagnoses. J Affect Disord 2004;78:131-40.

12 Awata S, Bech P, Yoshida S, et al. Reliability and validity of the Japanese version of the world health Organization-Five well-being index in the context of detecting depression in diabetic patients. Psychiatry Clin Neurosci 2007;61:112-9.

13 Tobin J. Estimation of relationships for limited dependent variables. Econometrica 1958;26:24-36.

14 Twisk JWR. Inleiding in de toegepaste biostatistiek. 3rd edn. Houten: Bohn Stafleu van Loghum, 2014.

15 Van Binsbergen JJ, Langens FNM, Dapper ALM, et al. NHGstandaard obesitas. Huisarts Wet 2010;53:609-25.

16 Dutch Diabetes Federation. NDF Zorgstandaard-diabetes type 2 volwassenen. Amersfoort: Dutch Diabetes Federation, 2015.

17 Adriaanse MC, Pouwer F, Dekker JM, et al. Diabetes-Related symptom distress in association with glucose metabolism and comorbidity: the Hoorn study. Diabetes Care 2008;31:2268-70.

18 Lee E-H, Lee K-W, Song R, et al. Psychometric evaluation of the Korean version of the diabetes symptom Checklist-Revised (DSC-R) for patients with type 2 diabetes. Health Qual Life Outcomes 2014;12:77

19 Wieringa TH, de Wit M, Twisk JWR, et al. Does hypoglycaemia affect the improvement in QOL after the transition to insulin in people with type 2 diabetes? J Endocrinol Invest 2018;41:249-58.

20 American Diabetes Association. Complications. Available: http:// www.diabetes.org/living-with-diabetes/complications/?loc= symptoms [Accessed 15 Oct 2018].

21 Gulliford MC, Mahabir D. Relationship of health-related quality of life to symptom severity in diabetes mellitus: a study in Trinidad and Tobago. J Clin Epidemiol 1999;52:773-80.

22 Van der Does FE, De Neeling JN, Snoek FJ, et al. Symptoms and well-being in relation to glycemic control in type II diabetes. Diabetes Care 1996;19:204-10.

23 Jensen Øystein, Bernklev T, Jelsness-Jørgensen L-P. Fatigue in type 1 diabetes: a systematic review of observational studies. Diabetes Res Clin Pract 2017;123:63-74.

24 Singh R, Teel C, Sabus C, et al. Fatigue in type 2 diabetes: impact on quality of life and predictors. PLoS One 2016;11:e0165652.

25 Menting J, Nikolaus S, van der Veld WM, et al. Severe fatigue in type 1 diabetes: Exploring its course, predictors and relationship with $\mathrm{HbA}_{\text {in }}$ in a prospective study. Diabetes Res Clin Pract 2016;121:127-34.

26 Von Korff M, Simon G. The relationship between pain and depression. Br J Psychiatry 1996;168:101-8.

27 Watson D, Pennebaker JW. Health complaints, stress, and distress: exploring the central role of negative affectivity. Psychol Rev 1989;96:234-54.

28 Teasdale JD. Negative thinking in depression: cause, effect, or reciprocal relationship? Advances in Behaviour Research and Therapy 1983;5:3-25.

29 Golub S. Periods: from menarche to menopause. 1st edn. Newbury Park: Sage Publications Inc, 1992.

30 McGlothlin AE, Lewis RJ. Minimal clinically important difference: defining what really matters to patients. JAMA 2014;312:1342-3.

31 Schafer JL, Graham JW. Missing data: our view of the state of the art. Psychol Methods 2002;7:147-77. 